\title{
A generalization of a 4-dimensional Einstein manifold
}

\author{
Yunhee Euh ${ }^{\dagger}$, JeongHyeong Park ${ }^{\ddagger}$ and Kouei Sekigawa ${ }^{\dagger}$ \\ ${ }^{\dagger}$ Department of Mathematics, Niigata University, Niigata 950-2181, JAPAN \\ $\ddagger$ Department of Mathematics, Sungkyunkwan University, Suwon 440-746, KOREA
}

\begin{abstract}
A weakly Einstein manifold is a generalization of a 4-dimensional Einstein manifold, which is defined as an application of a curvature identity derived from the generalized Gauss-Bonnet formula for a 4-dimensional compact oriented Riemannian manifold. In this paper, we shall give a characterization of a weakly Einstein manifold.
\end{abstract}

Mathematics Subsect Classification (2010) : 53B20, 53C20

Keywords :Einstein manifold, Singer-Thorpe basis

\section{Introduction}

In the previous paper [3], we derived a curvature identity on a 4-dimensional compact oriented Riemannian manifold from the generalized Gauss-Bonnet formula, and further gave a direct proof of the fact that the curvature identity holds on any 4-dimensional Riemannian manifold which is not necessarily compact. Consequently, we proved that the following curvature identity holds on any 4-dimensional Riemannian manifold $M=$ $(M, g)$ :

$$
\check{R}-2 \check{\rho}-L \rho+\tau \rho-\frac{1}{4}\left(|R|^{2}-4|\rho|^{2}+\tau^{2}\right) g=0 .
$$

Here,

$$
\begin{gathered}
\check{R}: \check{R}_{i j}=R_{a b c i} R^{a b c}, \quad \check{\rho}: \check{\rho}_{i j}=\rho_{a i} \rho^{a}{ }_{j}, \\
L:(L \rho)_{i j}=2 R_{i a b j} \rho^{a b},
\end{gathered}
$$

where $R, \rho$ and $\tau$ are the curvature tensor, the Ricci tensor and the scalar curvature of $M$, respectively.

From (1.1), we may easily check that any 4-dimensional Einstein manifold $M=(M, g)$ satisfies the condition

$$
R_{i}^{a b c} R_{a b c j}=\frac{1}{4}|R|^{2} g_{i j} .
$$

E-mail addresses: prettyfish@skku.edu (Y. Euh), parkj@skku.edu (J. H. Park), sekigawa@math.sc.niigata-u.ac.jp (K. Sekigawa). 
In [3], we defined a weakly Einstein manifold based on the above, i.e. Riemannian manifold $M=(M, g)$ satisfying the condition (1.2) (with $|R|^{2}$ not necessarily constant). By the definition, we see immediately that a weakly Einstein manifold in dimension 4 is a generalization of a 4-dimensional Einstein manifold (see Examples 4 and 5 in $\S 3$ ). We may also remark that a weakly Einstein manifold is not necessarily Einstein. As a characterization of a 4-dimensional Einstein manifold, the following theorem is wellknown.

Theorem A ([8]) A 4-dimensional Riemannian manifold $M=(M, g)$ is Einstein if and only if there exists a Singer-Thorpe basis of $T_{p} M$ at each point $p \in M$.

The main purpose of the present paper is to give a generalization of Theorem A. Namely, we shall prove the following:

Theorem B A 4-dimensional Riemannian manifold $M=(M, g)$ is weakly Einstein if and only if there exists a generalized Singer-Thorpe basis of $T_{p} M$ at each point $p \in M$.

In $\S 2$, we shall prepare some fundamental terminologies and notational conventions for the forthcoming arguments. In $\S 3$, we shall give a proof of Theorem B.

\section{Preliminaries}

Let $M=(M, g)$ be a 4-dimensional Riemannian manifold and $\mathfrak{X}(M)$ be the Lie algebra of all smooth vector fields on $M$. We denote the Levi-Civita connection, the curvature tensor, the Ricci tensor and the scalar curvature of $M$ by $\nabla, R, \rho$ and $\tau$, respectively. We assume that the curvature tensor $R$ is defined by $R(X, Y) Z=\left[\nabla_{X}, \nabla_{Y}\right] Z-\nabla_{[X, Y]} Z$ for $X, Y, Z \in \mathfrak{X}(M)$. Further, we denote the Ricci transformation by $Q$ given by $\rho(X, Y)=g(Q X, Y)$ for $X, Y \in \mathfrak{X}(M)$. Then, we may easily check that $Q$ is symmetric with respect to the metric $g$, namely, $g(Q X, Y)=g(X, Q Y)$ for $X, Y \in \mathfrak{X}(M)$. Now, we may rewrite the curvature identity (1.1) as follows:

$$
\begin{gathered}
\sum_{a, b, c} R_{a b c i} R_{a b c j}-2 \sum_{a} \rho_{a i} \rho_{a j}-2 \sum_{a, b} \rho_{a b} R_{i a b j} \\
+\tau \rho_{i j}-\frac{1}{4}\left(|R|^{2}-4|\rho|^{2}+\tau^{2}\right) \delta_{i j}=0
\end{gathered}
$$

with respect to an orthonormal basis $\left\{e_{i}\right\}(1 \leq i \leq 4)$ of $T_{p} M$ at any point $p \in M$, where $R_{i j k l}=g\left(R\left(e_{i}, e_{j}\right) e_{k}, e_{l}\right), \rho_{i j}=\rho\left(e_{i}, e_{j}\right)$.

We here introduce some special kinds of orthonormal basis of $T_{p} M(p \in M)$ and explain their intermediate relationships. We assume that an orthonormal basis $\left\{e_{i}\right\}(1 \leq i \leq 4)$ 
of $T_{p} M$ is simultaneously a Ricci eigenbasis and Chern basis [2, 6, 7] satisfying

$$
R_{1213}=R_{1214}=R_{1223}=R_{1224}=R_{1314}=R_{1323}=0 .
$$

Then, we have further

$$
R_{2434}=R_{2334}=R_{1434}=R_{1334}=R_{2324}=R_{1424}=0 .
$$

Thus, from (2.2) and (2.3), we have

$$
R_{i j j k}=0 \quad(i \neq k), \quad(1 \leq i, j, k \leq 4) .
$$

Conversely, if (2.4) holds with respect to an orthonormal basis $\left\{e_{i}\right\}$ of $T_{p} M$, then we see that the basis $\left\{e_{i}\right\}$ is a Ricci eigenbasis and a Chern basis at the same time.

The following example shows that a Ricci eigenbasis is not necessarily always a Chern basis.

Example 1 Let $\mathfrak{g}=\operatorname{span}_{\mathbb{R}}\left\{e_{1}, e_{2}, e_{3}, e_{4}\right\}$ be a 4-dimensional real Lie algebra equipped with the following Lie bracket operation:

$$
\begin{array}{llll}
{\left[e_{1}, e_{2}\right]=2 e_{2},} & {\left[e_{1}, e_{3}\right]=-e_{3},} & {\left[e_{1}, e_{4}\right]=2 e_{3}-e_{4},} \\
{\left[e_{2}, e_{3}\right]=0,} & {\left[e_{2}, e_{4}\right]=0,} & {\left[e_{3}, e_{4}\right]=0,}
\end{array}
$$

and $<,>$ the inner product on $\mathfrak{g}$ given by $\left.<e_{i}, e_{j}\right\rangle=\delta_{i j}$. Let $G$ be a connected and simply connected solvable Lie group with the Lie algebra $\mathfrak{g}$ of $G$ and $g$ the $G$-invariant Riemannian metric on $G$ determined by $<,>$. We set $\nabla_{e_{i}} e_{j}=\sum_{k=1}^{4} \Gamma_{i j k} e_{k},(1 \leq i, j \leq 4)$. Then, we get

$$
\Gamma_{i j k}=-\Gamma_{i k j}
$$

and further, from (2.5), we obtain

$$
\begin{aligned}
& \Gamma_{134}=-1, \quad \Gamma_{212}=-2, \quad \Gamma_{313}=1, \\
& \Gamma_{314}=-1, \quad \Gamma_{413}=-1, \quad \Gamma_{414}=1,
\end{aligned}
$$

and otherwise being zero up to sign. From (2.6) and (2.7), by direct calculations, we have

$$
\begin{array}{ll}
R_{1212}=4, & R_{1414}=4, \\
R_{2323}=-2, & R_{2424}=-2, \\
R_{1314}=-2, & R_{2324}=2,
\end{array}
$$

and otherwise being zero up to sign. Then, we have the Ricci eigenvalues as $\lambda_{1}=-8$, $\lambda_{2}=0, \lambda_{3}=2, \lambda_{4}=-2$. 
Now, we recall the definition of a Singer-Thorpe basis. An orthonormal basis $\left\{e_{i}\right\}$ of $T_{p} M$ $(p \in M)$ is called a Singer-Thorpe basis at $T_{p} M$ if the basis $\left\{e_{i}\right\}$ satisfies (2.4) and

$$
R_{1212}=R_{3434}, \quad R_{1313}=R_{2424}, \quad R_{1414}=R_{2323} .
$$

We here give a generalization of the Singer-Thorpe basis.

Definition 1 Let $M=(M, g)$ be a 4-dimensional Riemannian manifold and $\left\{e_{i}\right\}$ be an orthonormal basis of $T_{p} M$ at $p \in M$. If the basis $\left\{e_{i}\right\}$ satisfies (2.4) and

$$
R_{1212}^{2}=R_{3434}^{2}, \quad R_{1313}^{2}=R_{2424}^{2}, \quad R_{1414}^{2}=R_{2323}^{2},
$$

then the orthonormal basis is called a generalized Singer-Thorpe basis of $T_{p} M$.

\section{Proof of Theorem B}

First, we shall prove the following proposition which gives a necessary condition for a 4-dimensional Riemannian manifold to be weakly Einstein.

Proposition 3.1 Let $M=(M, g)$ be a weakly Einstein manifold and $\left\{e_{i}\right\}(1 \leq i \leq 4)$ an orthonormal Ricci eigenbasis of $T_{p} M$ corresponding to the eigenvalues $\lambda_{i}(1 \leq i \leq 4)$ at any point $p \in M$. Then, we see that the curvature condition

$$
R_{1212}{ }^{2}=R_{3434}{ }^{2}, \quad R_{1313}{ }^{2}=R_{2424}{ }^{2}, \quad R_{1414}{ }^{2}=R_{2323}{ }^{2}
$$

holds and also the following cases $(1) \sim(4)$ never occur:

(1) $\lambda_{1}=\lambda_{2}=\lambda_{3}(\neq 0), \lambda_{4}=0$,

(2) $\quad \lambda_{1}=\lambda_{2}=\lambda_{4}(\neq 0), \lambda_{3}=0$,

(3) $\lambda_{1}=\lambda_{3}=\lambda_{4}(\neq 0), \lambda_{2}=0$,

(4) $\lambda_{2}=\lambda_{3}=\lambda_{4}(\neq 0), \lambda_{1}=0$.

Especially, if $M$ is Einstein, then

$$
R_{1212}=R_{3434}, \quad R_{1313}=R_{2424}, \quad R_{1414}=R_{2323}
$$

holds for any orthonormal basis $\left\{e_{i}\right\}$ of $T_{p} M$.

Proof. Let $M=(M, g)$ be a weakly Einstein manifold and $p$ any point of $M$ and $\left\{e_{i}\right\}$ $(1 \leq i \leq 4)$ an orthonormal Ricci eigenbasis of $T_{p} M$ corresponding to the Ricci eigenvalues $\lambda_{i}(1 \leq i \leq 4)$ at $p$, namely, satisfying the following condition

$$
Q e_{i}=\lambda_{i} e_{i} \quad(1 \leq i \leq 4) .
$$


Then, from (3.2), we get

$$
\begin{aligned}
|R|^{2}= & 4\left\{R_{1212}{ }^{2}+R_{1313}^{2}+R_{1414}{ }^{2}+R_{2323}{ }^{2}+R_{2424}{ }^{2}+R_{3434}{ }^{2}\right. \\
& +4 R_{1213}{ }^{2}+4 R_{1214}{ }^{2}+4 R_{1223}{ }^{2}+4 R_{1224}{ }^{2}+4 R_{1314}{ }^{2}+4 R_{1323}{ }^{2} \\
& \left.+2 R_{1234}{ }^{2}+2 R_{1342}{ }^{2}+2 R_{1423}{ }^{2}\right\} .
\end{aligned}
$$

On the other hand, setting $i=j=1$ in the left hand side of (1.2), we get

$$
\begin{aligned}
\sum_{a, b, c} R_{a b c 1}{ }^{2}= & 2\left\{R_{1212}{ }^{2}+R_{1313}{ }^{2}+R_{1414}{ }^{2}+R_{1234}{ }^{2}+R_{1342}{ }^{2}+R_{1423}{ }^{2}\right. \\
& \left.+2\left(R_{1213}{ }^{2}+R_{1214}{ }^{2}+R_{1223}{ }^{2}+R_{1224}{ }^{2}+R_{1314}{ }^{2}+R_{1323}{ }^{2}\right)\right\} .
\end{aligned}
$$

From (3.3), (3.4), and taking account of (1.2), we have the following equality

$$
R_{1212}{ }^{2}+R_{1313}{ }^{2}+R_{1414}{ }^{2}-R_{2323}{ }^{2}-R_{2424}{ }^{2}-R_{3434}{ }^{2}=0 .
$$

Similarly, we get

$$
\begin{aligned}
& R_{1212}{ }^{2}+R_{2323}{ }^{2}+R_{2424}{ }^{2}-R_{1313}{ }^{2}-R_{1414}{ }^{2}-R_{3434}{ }^{2}=0, \\
& R_{1212}{ }^{2}+R_{1414}{ }^{2}+R_{2424}{ }^{2}-R_{1313}{ }^{2}-R_{2323}{ }^{2}-R_{3434}{ }^{2}=0, \\
& {R_{1212}}^{2}+R_{1313}{ }^{2}+R_{2323}{ }^{2}-R_{1414}{ }^{2}-R_{2424}{ }^{2}-R_{3434}{ }^{2}=0 .
\end{aligned}
$$

From (3.5) and (3.6), we have

$$
R_{1212}^{2}-R_{3434}^{2}=0 .
$$

Similarly, from (3.5) and (3.7), we have

$$
R_{1313}^{2}-R_{2424}^{2}=0 .
$$

From (3.5) and (3.8), we have

$$
R_{1414}^{2}-R_{2323}^{2}=0 .
$$

Thus, from (3.9) (3.11), we have (3.1).

Next, from (3.1), we see that the following eight cases can be taken into consideration;

Case (i) $R_{1212}=R_{3434}, R_{1313}=R_{2424}, R_{1414}=R_{2323}$.

Then, $\lambda_{1}-\lambda_{2}=0, \quad \lambda_{1}-\lambda_{3}=0, \quad \lambda_{1}-\lambda_{4}=0$, and hence, $\lambda_{1}=\lambda_{2}=\lambda_{3}=\lambda_{4}$.

Case (ii) $R_{1212}=-R_{3434}, R_{1313}=R_{2424}, R_{1414}=R_{2323}$.

Then, we get also

$$
\begin{array}{ll}
\lambda_{1}-\lambda_{2}=0, & \lambda_{1}-\lambda_{3}=-2 R_{1212}, \\
\lambda_{1}-\lambda_{4}=-2 R_{1212}, & \lambda_{2}-\lambda_{3}=-2 R_{1212}, \\
\lambda_{2}-\lambda_{4}=-2 R_{1212}, & \lambda_{3}-\lambda_{4}=0,
\end{array}
$$


and hence, $\lambda_{1}=\lambda_{2}, \lambda_{3}=\lambda_{4}$.

Case (iii) $R_{1212}=R_{3434}, R_{1313}=-R_{2424}, R_{1414}=R_{2323}$.

Then, we get

$$
\begin{array}{ll}
\lambda_{1}-\lambda_{2}=-2 R_{1313}, & \lambda_{1}-\lambda_{3}=0, \\
\lambda_{1}-\lambda_{4}=-2 R_{1313}, & \lambda_{2}-\lambda_{3}=2 R_{1313}, \\
\lambda_{2}-\lambda_{4}=0, & \lambda_{3}-\lambda_{4}=-2 R_{1313},
\end{array}
$$

and hence, $\lambda_{1}=\lambda_{3}, \lambda_{2}=\lambda_{4}$.

Case (iv) $R_{1212}=R_{3434}, R_{1313}=R_{2424}, R_{1414}=-R_{2323}$.

Then, we get

$$
\begin{array}{ll}
\lambda_{1}-\lambda_{2}=-2 R_{1414}, & \lambda_{1}-\lambda_{3}=-2 R_{1414}, \\
\lambda_{1}-\lambda_{4}=0, & \lambda_{2}-\lambda_{3}=0, \\
\lambda_{2}-\lambda_{4}=2 R_{1414}, & \lambda_{3}-\lambda_{4}=2 R_{1414},
\end{array}
$$

and hence, $\lambda_{1}=\lambda_{4}, \lambda_{2}=\lambda_{3}$.

Case (v) $R_{1212}=R_{3434}, R_{1313}=-R_{2424}, R_{1414}=-R_{2323}$.

Then, we get

$$
\begin{array}{ll}
\lambda_{1}+\lambda_{2}=-2 R_{1212}, & \lambda_{1}-\lambda_{3}=-2 R_{1414}, \\
\lambda_{1}-\lambda_{4}=-2 R_{1313}, & \lambda_{2}-\lambda_{3}=2 R_{1313}, \\
\lambda_{2}-\lambda_{4}=2 R_{1414}, & \lambda_{3}+\lambda_{4}=-2 R_{1212},
\end{array}
$$

and hence, $\lambda_{1}+\lambda_{2}=\lambda_{3}+\lambda_{4}$.

Case (vi) $R_{1212}=-R_{3434}, R_{1313}=R_{2424}, R_{1414}=-R_{2323}$.

Then, we get

$$
\begin{array}{ll}
\lambda_{1}-\lambda_{2}=-2 R_{1414}, & \lambda_{1}+\lambda_{3}=-2 R_{1313}, \\
\lambda_{1}-\lambda_{4}=-2 R_{1212}, & \lambda_{2}-\lambda_{3}=-2 R_{1212}, \\
\lambda_{2}+\lambda_{4}=-2 R_{1313}, & \lambda_{3}-\lambda_{4}=2 R_{1414},
\end{array}
$$

and hence, $\lambda_{1}+\lambda_{3}=\lambda_{2}+\lambda_{4}$.

Case (vii) $R_{1212}=-R_{3434}, R_{1313}=-R_{2424}, R_{1414}=R_{2323}$.

Then, we get

$$
\begin{array}{ll}
\lambda_{1}-\lambda_{2}=-2 R_{1313}, & \lambda_{1}-\lambda_{3}=-2 R_{1212}, \\
\lambda_{1}+\lambda_{4}=-2 R_{1414}, & \lambda_{2}+\lambda_{3}=-2 R_{1414}, \\
\lambda_{2}-\lambda_{4}=-2 R_{1212}, & \lambda_{3}-\lambda_{4}=-2 R_{1313},
\end{array}
$$

and hence, $\lambda_{1}+\lambda_{4}=\lambda_{2}+\lambda_{3}$.

Case (viii) $R_{1212}=-R_{3434}, R_{1313}=-R_{2424}, R_{1414}=-R_{2323}$. 
Then, we get

$$
\begin{array}{ll}
\lambda_{1}+\lambda_{2}=-2 R_{1212}, & \lambda_{1}+\lambda_{3}=-2 R_{1313}, \\
\lambda_{1}+\lambda_{4}=-2 R_{1414}, & \lambda_{2}+\lambda_{3}=2 R_{1414}, \\
\lambda_{2}+\lambda_{4}=2 R_{1313}, & \lambda_{3}+\lambda_{4}=2 R_{1212},
\end{array}
$$

and hence, $\lambda_{1}+\lambda_{2}+\lambda_{3}+\lambda_{4}=0$ (i.e., $\tau=0$ ).

Thus, from the above arguments in Cases $(\mathrm{i}) \sim($ viii $)$, we see that the cases $(1) \sim(4)$ in Proposition 3.1 do not occur.

Remark 1 In the proof of Proposition 3.1, we may note that Cases (ii) to (iv) (also for Cases (v) to (vii), respectively) are all essentially equivalent.

The following examples illustrate Proposition 3.1. Then, from the examples we can easily check that $M$ is not a weakly Einstein manifold.

Example 2 Let $M$ be a Riemannian product manifold of 2-dimensional Riemannian manifolds of constant Gaussian curvatures $c_{1}$ and $c_{2}$ satisfying $c_{1}^{2} \neq c_{2}^{2}$. Then this implies that $M$ is not a weakly Einstein manifold.

Example 3 Let $M=(M, g)$ be a Riemannian product manifold of a 3-dimensional space of constant sectional curvature $c(\neq 0)$ and a real line $\mathbb{R}$. From Proposition 3.1 , we see that $M$ is not a weakly Einstein manifold.

Remark 2 Based on Proposition 3.1 and the related Examples 2 and 3, it may be seen that the statement "for any 4-dimensional Riemannian manifold one always gets (1.2)" ([1], pp. 165), is incorrect.

The following examples show that a weakly Einstein manifold is not necessarily Einstein.

Example 4 ([3]) Let $M$ be a Riemannian product manifold of 2-dimensional Riemannian manifolds $M_{1}(c)$ and $M_{2}(-c)$ of constant Gaussian curvatures $c$ and $-c(c \neq 0)$, respectively. Then we can easily check that $M$ is not Einstein. We can also easily check that $M$ satisfies (1.2), thus $M$ is weakly Einstein. Further, $M$ belongs to Cases (ii), (vi), (vii) and (viii).

Example 5 Let $\mathfrak{g}=\operatorname{span}_{\mathbb{R}}\left\{e_{1}, e_{2}, e_{3}, e_{4}\right\}$ be a 4-dimensional real Lie algebra equipped with the following Lie bracket operation:

$$
\begin{aligned}
& {\left[e_{1}, e_{2}\right]=a e_{2}, \quad\left[e_{1}, e_{3}\right]=-a e_{3}-b e_{4}, \quad\left[e_{1}, e_{4}\right]=b e_{3}-a e_{4},} \\
& {\left[e_{2}, e_{3}\right]=0, \quad\left[e_{2}, e_{4}\right]=0, \quad\left[e_{3}, e_{4}\right]=0,}
\end{aligned}
$$


where $a(\neq 0), b$ are constant. We define an inner product $<,>$ on $\mathfrak{g}$ by

$<e_{i}, e_{j}>=\delta_{i j}$. Let $G$ be a connected and simply connected solvable Lie group with the Lie algebra $\mathfrak{g}$ of $G$ and $g$ the $G$-invariant Riemannian metric on $G$ determined by $<,>$. From (3.12),

$$
\Gamma_{134}=-b, \quad \Gamma_{212}=-a, \quad \Gamma_{313}=a, \quad \Gamma_{414}=a,
$$

and otherwise being zero up to sign. From (2.6) and (3.13), by direct calculations, we have

$$
\begin{array}{lll}
R_{1212}=a^{2}, & R_{1313}=a^{2}, & R_{1414}=a^{2} \\
R_{2323}=-a^{2}, & R_{2424}=-a^{2}, & R_{3434}=a^{2}
\end{array}
$$

and otherwise being zero up to sign. From this, we can easily check that $M$ is not Einstein since the Ricci curvature components satisfy $\rho_{11}=-3 a^{2}$ but $\rho_{22}=a^{2}$. We also can easily check that $M$ satisfies (1.2), thus $M$ is weakly Einstein. Then, we see that $(G, g)$ belongs to Case (v).

Remark 3 Jensen [5] proved that a 4-dimensional homogeneous Einstein manifold is locally symmetric. We may easily check that Example 5 is homogeneous but not locally symmetric. Thus, Example 5 shows that Jensen's result does not necessarily hold for weakly Einstein manifolds in general.

In the remainder of this section, we shall give a proof of Theorem B.

Necessity: From Proposition 3.1, it suffices to prove that there exists an orthonormal Ricci eigenbasis $\left\{e_{i}\right\}$ of $T_{p} M$ at each point $p \in M$ which satisfies (2.4). Let $M=(M, g)$ be a weakly Einstein manifold. Then, from (2.1), we have also the following equality

$$
2 \sum_{a} \rho_{a i} \rho_{a j}+2 \sum_{a, b} \rho_{a b} R_{i a b j}-\tau \rho_{i j}-|\rho|^{2} \delta_{i j}+\frac{\tau^{2}}{4} \delta_{i j}=0 .
$$

Setting $i=j=1$ in (3.15), we get

$$
2 \lambda_{1}^{2}+2 \sum_{i} \lambda_{i} R_{1 i i 1}-\left(\sum_{i} \lambda_{i}\right) \lambda_{1}-\sum_{i} \lambda_{i}^{2}+\frac{1}{4}\left(\sum_{i} \lambda_{i}\right)^{2}=0 .
$$

Similarly, we get

$$
\begin{aligned}
& 2 \lambda_{2}^{2}+2 \sum_{i} \lambda_{i} R_{2 i i 2}-\left(\sum_{i} \lambda_{i}\right) \lambda_{2}-\sum_{i} \lambda_{i}^{2}+\frac{1}{4}\left(\sum_{i} \lambda_{i}\right)^{2}=0, \\
& 2 \lambda_{3}^{2}+2 \sum_{i} \lambda_{i} R_{3 i i 3}-\left(\sum_{i} \lambda_{i}\right) \lambda_{3}-\sum_{i} \lambda_{i}^{2}+\frac{1}{4}\left(\sum_{i} \lambda_{i}\right)^{2}=0, \\
& 2 \lambda_{4}^{2}+2 \sum_{i} \lambda_{i} R_{4 i i 4}-\left(\sum_{i} \lambda_{i}\right) \lambda_{4}-\sum_{i} \lambda_{i}^{2}+\frac{1}{4}\left(\sum_{i} \lambda_{i}\right)^{2}=0 .
\end{aligned}
$$


Further, setting $i=1, j=2$ in (3.15), we get the following

$$
\left(\lambda_{3}-\lambda_{4}\right) R_{1323}=0 .
$$

Similarly, we get

$$
\begin{array}{ll}
\left(\lambda_{2}-\lambda_{4}\right) R_{1223}=0, & \left(\lambda_{2}-\lambda_{3}\right) R_{1224}=0, \quad\left(\lambda_{1}-\lambda_{4}\right) R_{1213}=0, \\
\left(\lambda_{1}-\lambda_{3}\right) R_{1214}=0, & \left(\lambda_{1}-\lambda_{2}\right) R_{1314}=0 .
\end{array}
$$

Then, the following cases are considerable:

Case I $\lambda_{1}=\lambda_{2}=\lambda_{3}=\lambda_{4}$.

Case II-1 $\lambda_{1}=\lambda_{2}(\equiv \lambda), \quad \lambda_{3} \neq \lambda_{4}, \quad\left(\lambda_{3}, \lambda_{4} \neq \lambda\right)$.

Case II-2 $\lambda_{1}=\lambda_{3}(\equiv \lambda), \quad \lambda_{2} \neq \lambda_{4}, \quad\left(\lambda_{2}, \lambda_{4} \neq \lambda\right)$.

Case II-3 $\lambda_{1}=\lambda_{4}(\equiv \lambda), \quad \lambda_{2} \neq \lambda_{3}, \quad\left(\lambda_{2}, \lambda_{3} \neq \lambda\right)$.

Case II-4 $\lambda_{2}=\lambda_{3}(\equiv \lambda), \quad \lambda_{1} \neq \lambda_{4}, \quad\left(\lambda_{1}, \lambda_{4} \neq \lambda\right)$.

Case II-5 $\lambda_{2}=\lambda_{4}(\equiv \lambda), \quad \lambda_{1} \neq \lambda_{3}, \quad\left(\lambda_{1}, \lambda_{3} \neq \lambda\right)$.

Case II-6 $\lambda_{3}=\lambda_{4}(\equiv \lambda), \quad \lambda_{1} \neq \lambda_{2}, \quad\left(\lambda_{1}, \lambda_{2} \neq \lambda\right)$.

Case III-1 $\lambda_{1}=\lambda_{2}(\equiv \lambda), \quad \lambda_{3}=\lambda_{4}(\equiv \mu), \quad(\lambda \neq \mu)$.

Case III-2 $\lambda_{1}=\lambda_{3}(\equiv \lambda), \quad \lambda_{2}=\lambda_{4}(\equiv \mu), \quad(\lambda \neq \mu)$.

Case III-3 $\lambda_{1}=\lambda_{4}(\equiv \lambda), \quad \lambda_{2}=\lambda_{3}(\equiv \mu), \quad(\lambda \neq \mu)$.

Case IV-1 $\lambda_{1}=\lambda_{2}=\lambda_{3}(\equiv \lambda), \quad \lambda_{4} \neq \lambda$.

Case IV-2 $\lambda_{1}=\lambda_{2}=\lambda_{4}(\equiv \lambda), \quad \lambda_{3} \neq \lambda$.

Case IV-3 $\lambda_{1}=\lambda_{3}=\lambda_{4}(\equiv \lambda), \quad \lambda_{2} \neq \lambda$.

Case IV-4 $\lambda_{2}=\lambda_{3}=\lambda_{4}(\equiv \lambda), \quad \lambda_{1} \neq \lambda$.

Case V $\quad \lambda_{i} \neq \lambda_{j}, \quad(i \neq j)$.

Case I. The existence of a generalized Singer-Thorpe basis follows immediately from the construction of a Singer-Thorpe basis.

Case V. Then, from (3.18) and (3.19), we may immediately choose a generalized SingerThorpe basis.

Case II-1. Then, it suffices to consider Cases (v) and (viii). First, we deal with Case (v). From (3.18) and (3.19), taking account of the equalities in Case (v), we have

$$
\begin{aligned}
& R_{1323}=0, R_{1223}=0, R_{1224}=0, \\
& R_{1213}=0, R_{1214}=0, R_{1313}=R_{2323} .
\end{aligned}
$$

Here, we note that all of the relations in (3.20) and Case (v) are preserved under the changes of the orthonormal basis satisfying the conditions of Case II-1. We denote the 2-dimensional subspace of $T_{p} M$ spanned $\left\{e_{1}, e_{2}\right\}$ by $V$. For any non-zero vector $x \in V$, 
we denote by $x^{\perp}$ the vector in $V$ such that $\left|x^{\perp}\right|=|x|, g\left(x, x^{\perp}\right)=0$, and the ordered pair $\left\{x, x^{\perp}\right\}$ and $\left\{e_{1}, e_{2}\right\}$ determine the same orientation on $V$. We define a unit vector $e \in V$ by

$$
R\left(e, e_{3}, e^{\perp}, e_{4}\right)=\max _{x \in V,|x|=1} R\left(x, e_{3}, x^{\perp}, e_{4}\right) .
$$

We set $e_{1}^{\prime}=e, e_{2}^{\prime}=e^{\perp}, e_{3}^{\prime}=e_{3}, e_{4}^{\prime}=e_{4}$ and define a function $\phi(t)$ by

$$
\phi(t)=R\left(\cos t e_{1}^{\prime}+\sin t e_{2}^{\prime}, e_{3}^{\prime},-\sin t e_{1}^{\prime}+\cos t e_{2}^{\prime}, e_{4}^{\prime}\right) .
$$

Then, from (3.21) and (3.22), we have $\phi^{\prime}(0)=0$, and hence,

$$
0=-R_{1314}^{\prime}+R_{2324}^{\prime}=-2 R_{1314}^{\prime}\left(\text { and hence, } R_{2324}^{\prime}=0\right. \text { ), }
$$

where $R_{i j k l}^{\prime}=R\left(e_{i}^{\prime}, e_{j}^{\prime}, e_{k}^{\prime}, e_{l}^{\prime}\right), 1 \leq i, j, k, l \leq 4$. Then together with (3.23), the respective equalities in (3.20) and Case (v) corresponding to the orthonormal basis $\left\{e_{i}^{\prime}\right\}$, we see that the orthonormal basis $\left\{e_{i}^{\prime}\right\}$ is a generalized Singer-Thorpe basis. Similarly, we may also choose a generalized Singer-Thorpe basis for Case (viii). Further, we may also choose a generalized Singer-Thorpe basis for Cases II-2 II-6.

Case III-1. Then it suffices to consider Cases (ii), (vi), (vii), (viii). First, we consider Case (ii). Then, from (3.18) and (3.19), we have

$$
R_{1223}=0, R_{1224}=0, R_{1213}=0, R_{1214}=0 .
$$

Here, we may note that each of the relations in (3.24) and Case (ii) is preserved under the changes of the orthonormal basis satisfying the conditions of Case III-1. Let $V$ be a 2dimensional subspace of $T_{p} M$ spanned by $\left\{e_{1}, e_{2}\right\}$ and $V^{\perp}$ be the orthogonal complement of $V$ in $T_{p} M$. Then $V^{\perp}$ is spanned by $\left\{e_{3}, e_{4}\right\}$. We define $e_{1}^{\prime} \in V$ and $e_{3}^{\prime} \in V^{\perp}$ by

$$
R\left(e_{1}^{\prime}, e_{3}^{\prime}, e_{1}^{\prime}, e_{3}^{\prime}\right)=\max _{\substack{x \in V, y \in V^{\perp} \\|x|=|y|=1}} R(x, y, x, y) .
$$

Further, we choose unit vectors $e_{2}^{\prime} \in V$ and $e_{4}^{\prime} \in V^{\perp}$ in such a way that $\left\{e_{1}, e_{2}\right\}$ and $\left\{e_{1}^{\prime}, e_{2}^{\prime}\right\} \quad\left(\left\{e_{3}, e_{4}\right\}\right.$ and $\left.\left\{e_{3}^{\prime}, e_{4}^{\prime}\right\}\right)$ define the same orientation on $V$ (on $V^{\perp}$, respectively). We define the function $\phi(t)$ by

$$
\phi(t)=R\left(e_{1}^{\prime}, \cos t e_{3}^{\prime}+\sin t e_{4}^{\prime}, e_{1}^{\prime}, \cos t e_{3}^{\prime}+\sin t e_{4}^{\prime}\right) .
$$

Then, we have $\phi^{\prime}(0)=0$, and hence

$$
R_{1314}^{\prime}=0
$$


Similarly, considering the function $\psi(t)$ defined by

$$
\psi(t)=R\left(\cos t e_{1}^{\prime}+\sin t e_{2}^{\prime}, e_{3}^{\prime}, \cos t e_{1}^{\prime}+\sin t e_{2}^{\prime}, e_{3}^{\prime}\right),
$$

we have $\psi^{\prime}(0)=0$, and hence,

$$
R_{1323}^{\prime}=0 .
$$

Then, from (3.24), (3.26) and (3.27), we see that the orthonormal basis $\left\{e_{i}^{\prime}\right\}$ is a generalized Singer-Thorpe basis. Similarly to Case (ii), we may choose a generalized SingerThorpe basis for Cases (vi), (vii), (viii). Further, we may also choose a generalized Singer-Thorpe basis for Cases III-2 and III-3.

Case IV-1. Then, it suffices to consider Case (viii) with $\lambda \neq 0$. Then from (3.18) and (3.19), we have

$$
R_{1223}=0, R_{1213}=0, R_{1323}=0 .
$$

Further, from Case (viii), we have

$$
\begin{aligned}
& R_{1212}=R_{1313}=R_{2323}=-\lambda, \\
& R_{1414}=R_{2424}=R_{3434}=\lambda .
\end{aligned}
$$

Here, we note that each of the relations in (3.28) and (3.29) is preserved under the changes of the orthonormal basis satisfying the conditions of Case IV-1. Let $V$ be a 3-dimensional subspace of $T_{p} M$ spanned by $\left\{e_{1}, e_{2}, e_{3}\right\}$ satisfying that $V$ is orthogonal complement of $\left\{e_{4}\right\}$. We define

$$
R\left(e_{1}^{\prime}, e_{2}^{\prime}, e_{2}^{\prime}, e_{4}^{\prime}\right)=\max _{\substack{x, y \in V, x \perp y \\|x|=|y|=1}} R\left(x, y, y, e_{4}\right),
$$

where $e_{3}^{\prime} \in V$ such that $e_{3}^{\prime} \perp e_{1}^{\prime}, e_{3}^{\prime} \perp e_{2}^{\prime},\left|e_{3}^{\prime}\right|=1, e_{4}^{\prime}=e_{4}$. First, we define the function $\phi(t)$ by

$$
\phi(t)=R\left(e_{1}^{\prime}, \cos t e_{2}^{\prime}+\sin t e_{3}^{\prime}, \cos t e_{2}^{\prime}+\sin t e_{3}^{\prime}, e_{4}^{\prime}\right) .
$$

Then, by the hypothesis (3.30), we have $\phi^{\prime}(0)=0$, and hence,

$$
R_{1234}^{\prime}+R_{1324}^{\prime}=0 .
$$

Next, we consider the function $\psi(t)$ defined by

$$
\psi(t)=R\left(\cos t e_{1}^{\prime}+\sin t e_{3}^{\prime}, e_{2}^{\prime}, e_{2}^{\prime}, e_{4}^{\prime}\right) .
$$

Then we have $0=\psi^{\prime}(0)=R_{3224}^{\prime}$, and hence,

$$
R_{1314}^{\prime}=0
$$


Next, we consider the function $\zeta(t)$ defined by

$$
\zeta(t)=R\left(\cos t e_{1}^{\prime}+\sin t e_{2}^{\prime},-\sin t e_{1}^{\prime}+\cos t e_{2}^{\prime},-\sin t e_{1}^{\prime}+\cos t e_{2}^{\prime}, e_{4}^{\prime}\right)
$$

Then, by the hypothesis we have also $0=\zeta^{\prime}(0)=-R_{1214}^{\prime}$, and hence

$$
R_{1214}^{\prime}=0
$$

Now, we set

$$
\begin{gathered}
e_{2}^{\prime \prime}=\frac{1}{\sqrt{2}} e_{2}^{\prime}+\frac{1}{\sqrt{2}} e_{3}^{\prime}, \\
e_{3}^{\prime \prime}=-\frac{1}{\sqrt{2}} e_{2}^{\prime}+\frac{1}{\sqrt{2}} e_{3}^{\prime}, \\
e_{1}^{\prime \prime}=e_{1}^{\prime}, e_{4}^{\prime \prime}=e_{4}^{\prime} .
\end{gathered}
$$

Then, we have

$$
\begin{aligned}
R\left(e_{1}^{\prime \prime}, e_{2}^{\prime \prime}, e_{2}^{\prime \prime}, e_{4}^{\prime \prime}\right) & =\frac{1}{2} R\left(e_{1}^{\prime}, e_{2}^{\prime}+e_{3}^{\prime}, e_{2}^{\prime}+e_{3}^{\prime}, e_{4}^{\prime}\right) \\
& =\frac{1}{2}\left\{R_{1224}^{\prime}+R_{1324}^{\prime}+R_{1234}^{\prime}+R_{1334}^{\prime}\right\}=0
\end{aligned}
$$

by virtue of (3.32), and hence,

$$
R_{1224}^{\prime \prime}=0 \text {. }
$$

Here, we set $R_{i j k l}^{\prime \prime}=R\left(e_{i}^{\prime \prime}, e_{j}^{\prime \prime}, e_{k}^{\prime \prime}, e_{l}^{\prime \prime}\right), 1 \leq i, j, k, l \leq 4$. Similarly, from (3.37), we have

$$
\begin{array}{r}
R_{1214}^{\prime \prime}=\frac{1}{\sqrt{2}} R\left(e_{1}^{\prime}, e_{2}^{\prime}+e_{3}^{\prime}, e_{1}^{\prime}, e_{4}^{\prime}\right)=\frac{1}{\sqrt{2}}\left(R_{1214}^{\prime}+R_{1314}^{\prime}\right)=0, \\
R_{1314}^{\prime \prime}=\frac{1}{\sqrt{2}} R\left(e_{1}^{\prime},-e_{2}^{\prime}+e_{3}^{\prime}, e_{1}^{\prime}, e_{4}^{\prime}\right)=\frac{1}{\sqrt{2}}\left(-R_{1214}^{\prime}+R_{1314}^{\prime}\right)=0
\end{array}
$$

by virtue of (3.34) and (3.36). Thus, from (3.38) and (3.39), we see that the orthonormal basis $\left\{e_{i}^{\prime \prime}\right\}$ is a generalized Singer-Thorpe basis. Similarly, we may also choose a generalized Singer-Thorpe basis for Cases IV-2 IV-4.

Sufficiency: We assume that $M=(M, g)$ admits a generalized Singer-Thorpe basis $\left\{e_{i}\right\}$. From the condition (2.4), we see that (3.18) and (3.19) hold on $M$. Further, by substituting $\lambda_{i}=\sum_{k} R_{i k k i}(1 \leq i \leq 4)$ to the left hand sides of (3.16) and (3.17), and taking account of (3.1), we see also that each equation in (3.16) and (3.17) holds. Therefore we see that $M$ satisfies the curvature condition (3.15). Thus $M$ is a weakly Einstein manifold by virtue of (2.1). This completes the proof of Theorem B. 


\section{An application}

In this section, we shall give a generalization of the Hitchin inequality for a 4-dimensional compact oriented Einstein manifold. Let $M=(M, g)$ be a compact oriented weakly Einstein manifold. Then, from Theorem B, we may choose an generalized Singer-Thorpe basis $\left\{e_{i}\right\}$ of $T_{p} M$ at any point $p \in M$ compatible with the orientation of $M$. We set

$$
\begin{array}{lll}
\alpha_{1}^{\prime}=R_{1212}, & \alpha_{2}^{\prime}=R_{1313}, & \alpha_{3}^{\prime}=R_{1414}, \\
\alpha_{1}^{\prime \prime}=R_{3434}, & \alpha_{2}^{\prime \prime}=R_{2424}, & \alpha_{3}^{\prime \prime}=R_{2323}, \\
\beta_{1}=R_{1234}, & \beta_{2}=R_{1342}, & \beta_{3}=R_{1423} .
\end{array}
$$

Then, from (4.1), by the first Bianchi identity,

$$
\beta_{1}+\beta_{2}+\beta_{3}=0
$$

Further, we set $\mathbf{a}^{\prime}=\left(\alpha_{1}^{\prime}, \alpha_{2}^{\prime}, \alpha_{3}^{\prime}\right)$, $\mathbf{a}^{\prime \prime}=\left(\alpha_{1}^{\prime \prime}, \alpha_{2}^{\prime \prime}, \alpha_{3}^{\prime \prime}\right)$ and $\mathbf{b}=\left(\beta_{1}, \beta_{2}, \beta_{3}\right)$ and denote the canonical inner product by $<,>$ on the 3 -dimensional Euclidean space $\mathbb{R}^{3}$. We set $|\mathbf{x}|=\sqrt{\langle\mathbf{x}, \mathbf{x}\rangle}$ for any $\mathbf{x} \in \mathbb{R}^{3}$. Then we may note that $\left|\mathbf{a}^{\prime}\right|=\left|\mathbf{a}^{\prime \prime}\right|$ by virtue of (2.10). Now, we denote the Euler number and the first Pontrjagin number of $M$ by $\chi(M)$ and $p_{1}(M)$, respectively. Then, from (4.1), applying the similar arguments in [4], we have the following equalities:

$$
\chi(M)=\frac{1}{4 \pi^{2}} \int_{M}\left\{<\mathbf{a}^{\prime}, \mathbf{a}^{\prime \prime}>+|\mathbf{b}|^{2}\right\} d v_{g}
$$

and

$$
p_{1}(M)=\frac{1}{2 \pi^{2}} \int_{M}<\mathbf{a}^{\prime}+\mathbf{a}^{\prime \prime}, \mathbf{b}>d v_{g},
$$

where $d v_{g}$ is the volume element of $M$. Now, we set

$$
\mathbf{a}=\frac{1}{2}\left(\mathbf{a}^{\prime}+\mathbf{a}^{\prime \prime}\right)
$$

Then, by (4.5), the equalities (4.3) and (4.4) are rewritten respectively by

$$
\begin{gathered}
\chi(M)=\frac{1}{4 \pi^{2}} \int_{M}\left\{2|\mathbf{a}|^{2}-\left|\mathbf{a}^{\prime}\right|^{2}+|\mathbf{b}|^{2}\right\} d v_{g} \\
p_{1}(M)=\frac{1}{2 \pi^{2}} \int_{M} 2<\mathbf{a}, \mathbf{b}>d v_{g}
\end{gathered}
$$


Then, from (4.6) and (4.7), we have the following:

$$
\begin{aligned}
& 2 \chi(M) \pm p_{1}(M) \\
= & \frac{1}{2 \pi^{2}} \int_{M}\left\{|\mathbf{a}|^{2}+|\mathbf{b}|^{2} \pm 2<\mathbf{a}, \mathbf{b}>+|\mathbf{a}|^{2}-\left|\mathbf{a}^{\prime}\right|^{2}\right\} d v_{g} \\
= & \frac{1}{2 \pi^{2}} \int_{M}\left\{|\mathbf{a} \pm \mathbf{b}|^{2}+|\mathbf{a}|^{2}-\left|\mathbf{a}^{\prime}\right|^{2}\right\} d v_{g} \\
\geq & \frac{1}{2 \pi^{2}} \int_{M}\left\{|\mathbf{a}|^{2}-\left|\mathbf{a}^{\prime}\right|^{2}\right\} d v_{g} .
\end{aligned}
$$

We set $f=|\mathbf{a}|^{2}-\left|\mathbf{a}^{\prime}\right|^{2}$, Then, from the definition of the vectors $\mathbf{a}^{\prime}$, $\mathbf{a}^{\prime \prime}$ and $\mathbf{a}$, taking account of the proof of Proposition 3.1, we have

$$
\begin{array}{ccc}
f=0 & \text { for Case (i), } \\
f=-\frac{1}{4}\left(\lambda_{1}-\lambda_{3}\right)^{2} \quad\left(\lambda_{1}=\lambda_{2}, \lambda_{3}=\lambda_{4}\right) & \text { for Case (ii), } \\
f=-\frac{1}{4}\left(\lambda_{1}-\lambda_{2}\right)^{2} \quad\left(\lambda_{1}=\lambda_{3}, \lambda_{2}=\lambda_{4}\right) & \text { for Case (iii), } \\
f=-\frac{1}{4}\left(\lambda_{1}-\lambda_{3}\right)^{2} \quad\left(\lambda_{1}=\lambda_{4}, \lambda_{2}=\lambda_{3}\right) & \text { for Case (iv), } \\
f=-\frac{1}{4}\left\{\left(\lambda_{1}-\lambda_{3}\right)^{2}+\left(\lambda_{1}-\lambda_{4}\right)^{2}\right\} \quad\left(\lambda_{1}+\lambda_{2}=\lambda_{3}+\lambda_{4}\right) & \text { for Case (v), } \\
f=-\frac{1}{4}\left\{\left(\lambda_{1}-\lambda_{2}\right)^{2}+\left(\lambda_{1}-\lambda_{4}\right)^{2}\right\} \quad\left(\lambda_{1}+\lambda_{3}=\lambda_{2}+\lambda_{4}\right) & \text { for Case (vi), } \\
f=-\frac{1}{4}\left\{\left(\lambda_{1}-\lambda_{2}\right)^{2}+\left(\lambda_{1}-\lambda_{3}\right)^{2}\right\} & \left(\lambda_{1}+\lambda_{4}=\lambda_{2}+\lambda_{3}\right) & \text { for Case (vii), } \\
f=-\frac{1}{4}\left\{\left(\lambda_{1}+\lambda_{2}\right)^{2}+\left(\lambda_{1}+\lambda_{3}\right)^{2}+\left(\lambda_{1}+\lambda_{4}\right)^{2}\right\} &
\end{array}
$$

at $p \in M$. Then from (4.10) (4.16), we see that $f$ gives rise a continuous function on $M$ and further, $f=0$ holds at $p$ if and only if $\lambda_{1}=\lambda_{2}=\lambda_{3}=\lambda_{4}$ holds at $p$ (namely, $M$ is Einstein at $p$ ). Therefore, summing up the above arguments we have finally the following Theorem.

Theorem C Let $M=(M, g)$ be a compact weakly Einstein manifold. Then, the following inequality holds on $M$ :

$$
2 \chi(M) \pm p_{1}(M) \geq C,
$$

where $C=\frac{1}{2 \pi^{2}} \int_{M}\left\{|\mathbf{a}|^{2}-\left|\mathbf{a}^{\prime}\right|^{2}\right\} d v_{g} \leq 0$. 
Remark 4 Since $p_{1}(M)=3 \sigma(M)(\sigma(M)$ is the Hirzebruch signature of $M)$, from Theorem $\mathrm{C}$ together with the proof, we see that the inequality (4.17) reduces to the Hitchin inequality [4]

$$
2 \chi(M) \geq 3|\sigma(M)|
$$

for the case where $M$ is Einstein. Thus, the inequality (4.17) in Theorem $\mathrm{C}$ is regarded as the generalization of the Hitchin inequality (4.18).

The following example illustrates Theorem $\mathrm{C}$ and Remark 4 .

Example 6 Let $M_{1}$ and $M_{2}$ be a unit 2-sphere and a compact oriented surface of genus $m(m \geq 2)$ with constant Gaussian curvature -1 , respectively, and further, $M$ be the Riemannian product of $M_{1}$ and $M_{2}, M=M_{1} \times M_{2}$. Then, we may easily check that $M$ is a compact, oriented weakly Einstein manifold which is a special case of Example 4, Then, by taking account of the Künneth formula, the Gauss-Bonnet formula and the formulas in [4], we have

$$
\left.\chi(M)=4(1-m), p_{1}(M)=0 \text { (thus, } \sigma(M)=0\right) \text {, and } C=8(1-m) .
$$

Therefore, from (4.19), we see that the equality sign of the inequality (4.17) in Theorem C holds for $M$, but $M$ does not satisfy the Hitchin inequality (4.18).

\section{Acknowledgements}

Research of Yunhee Euh was supported by the National Research Foundation of Korea Grant funded by the Korean Government [NRF-2009-352-C00007]. Research of JeongHyeong Park was supported by Basic Science Research Program through the National Research Foundation of Korea(NRF) funded by the Ministry of Education, Science and Technology (2009-0087201).

\section{References}

[1] A. L. Besse, Manifolds all of whose geodesics are closed, Ergeb. Math. Grenzgeb. 93, Springer-Verlag, Berlin, Heidelberg, New York, 1978.

[2] S. S. Chern, On the Curvature and Characteristic Classes of a Riemannian manifold, Abh. Math. Sem. Hamburg 20 (1955), 117-126. 
[3] Y. Euh, J. H. Park and K. Sekigawa, A Curvature identity on a 4-dimensional Riemannian manifold, arXiv:1008.2439.

[4] N. Hitchin, Compact four-diemensional Einstein manifolds, J. Differential Geometry 9 (1974), 435-441.

[5] G. R. Jensen, Homogeneous Einstein spaces of dimension four, J. Differential Geometry 3 (1969), 309-349.

[6] R. Klinger, A Basis that Reduces to Zero as many Curvature Components as Possible, Abh. Math. Sem. Univ. Hamburg 61 (1991), 243-248.

[7] O. Kowalski and F. Prüfer, Curvature tensors in dimension four which do not belong to any curvature homogeneous space, Archivum Mathematicum (Brno) Tomus 30 (1994), 45-57.

[8] I. M. Singer and J. A. Thorpe, The curvature of 4-dimensional Einstein spaces, Global Analysis, (Papers in Honor of K. Kodaira) 355-365 Univ. Tokyo Press, Tokyo. 